Beilstein u. Kupffer, über Wermuthöl.

\begin{tabular}{cccc} 
& & & \multicolumn{2}{c}{ Gefunden } \\
\cline { 3 - 4 } $2 \mathrm{H}_{2} \mathrm{O}$ & 7,2 & 7,6 & 6,7 \\
$\mathrm{Ca}$ & 8,6 & 8,2 & $8,2$.
\end{tabular}

Das Krystallwasser entweicht nicht über Schwefelsäure.

Eine genaue krystallographische Untersuchung der Krystalle des Kalksalzes von verschiedener Herkunft, von Herrn M. J er of e jew ausgeführt, beweist die vollkommene Uebereinstimmung derselben. Angesichts dieser Thatsachen halten wir die völlige Identität des Cymols aus Campher und aus Kümmelöl für erwiesen.

\title{
Ueber Wermuthöl;
} von Denselben.

Nach F. Lebla n c*) enthält das Oel aus dem blühenden Kraut von Artemisia Absinthium L. als Hauptbestandtheil ein Oel $\mathrm{C}_{10} \mathrm{H}_{16} \mathrm{O}$ von der Zusammensetzung des Camphers, welches mit Phosphorsäureanhydrid den Kohlenwasserstoff $\mathrm{C}_{\mathbf{1 0}} \mathrm{H}_{\mathbf{1 4}}$ liefert. Dieses Resultat ist von $\mathrm{Cah}$ ou $\mathrm{rs}^{* *}$ ), Glads t on $\mathrm{e}^{* * *}$ ) und Schwaner $t+$ ) bestätigt worden. Gladst on e fand aufserdem im Wermuthöl einen Kohlenwasserstoff und ein tiefblaues Del (Cöruleinn, Azulen $\mathrm{C}_{16} \mathrm{H}_{24} \cdot \mathrm{H}_{2} \mathrm{O}$ nach $\mathrm{P}$ iesse $+\mathrm{t}$ ), das er als identisch mit dem blauen Körper aus Kamillen- und Schafgarbenöl betrachtet. Um dic Natur des von Leblanc beobachteten Kohlenwasserstoffs $\mathrm{C}_{10} \mathrm{H}_{14}$ kennen zu lernen

*) Gmelin's Handbuch [1] ₹, 326.

**) Compt. rend. 25,725 .

***) Jahresbericht für Chemie u. s. w. für $1863,549$.

f) Diese Annalen 128, 110.

†) Jahresbericht für Chemie u. s. w. für 1863, 549 . 
unterwarfen wir das Wermuthöl einer fractionirten Destillation. Wir fanden hierbei die Angaben von Glad s to ne vollkommen bestätigt.

Der unter $160^{\circ}$ siedende Antheil des Wermuthöls ist ein Terpen $\mathrm{C}_{10} \mathrm{H}_{16}$, das übrigens hartnäckig noch Theile des sauerstoffhaltigen Oeles zurückhält, selbst nach dem Rectificiren über Natrium.

Der Haupttheil des Wermuthöles siedete constant bei $\mathbf{1 9 5}^{\circ}$, während Leblanc den Siedepunkt $204^{\circ}$ angiebt. Die von Leblanc angegebene Zusammensetzung des Oeles $\mathrm{C}_{10} \mathrm{H}_{16} \mathrm{O}$ hat Schwanert bestätigt. Unsere Analysen ergaben ein etwas abweichendes Resultat, doch halten wir die angenommene Formel für richtig, da eine andere wahrscheinliche Formel sich aus unseren Analysen nicht berechnen läfst. Vermuthlich war unser Product noch durch einen sauerstoffhaltigen Körper verunreinigt, von dem wir es durch blofses Fractioniren nicht reinigen konnten. Die Bildung von Cymol aus dem Körper läfst kaum einen Zweifel über die Zusammensetzung des Oeles. Wir wollen es Absinthal nennen.

1. $0,3104 \mathrm{Grm}$. von 194 bis $196^{\circ}$ siedend gaben $0,8738 \mathrm{CO}_{2}$ und $0,3193 \mathrm{H}_{2} \mathrm{O}$.

2. $0,3417 \mathrm{Grm}$. von 194 bis $196^{\circ}$ siedend gaben $0,9643 \mathrm{CO}_{2}$ und $0,3535 \mathrm{H}_{2} \mathrm{O}$.

3. 0,4205 Grm. gaben $0,4250 \mathrm{H}_{2} \mathrm{O}$ and $1,1652 \mathrm{CO}_{2}$.

\begin{tabular}{|c|c|c|c|c|}
\hline & Berechnet & & efund & \\
\hline & $\mathrm{C}_{10} \mathrm{H}_{16} \mathrm{O}$ & 1. & 2. & 3. \\
\hline $\mathrm{C}_{10}$ & 78,9 & 76,8 & 77,0 & 75,6 \\
\hline $\mathrm{H}_{16}$ & 10,5 & 11,4 & $11, \tilde{5}$ & 11,2 \\
\hline 0 & 10,6 & - & - & - \\
\hline & $100,0$. & & & \\
\hline
\end{tabular}

Nach Schwanert giebt der bei $204^{0}$ siedende Antheil des Wermuthöles keine Camphersäure, wohl aber Camphresinsäure. Salpetersäure vom spec. Gewicht 1,27 wirkt sehr lebhaft auf Absinthol ein. Eine verdünntere Säure (1 Vol. $\mathrm{NHO}_{3}$ spec. Gewicht 1,27 und $2 \mathrm{Vol} . \mathrm{H}_{2} \mathrm{O}$ ) wirkt ruhiger ein. 
Es bildet sich viel Harz, aber keine Camphersäure. Nebenbei erhielten wir kleine Mengen einer in Wasser sehr schwerlöslichen Säure von wenig charakteristischen Eigenschaften. Wir haben sie nicht weiter verfolgt.

Beim Schmelzen von Absinthol mit Aetzkali bildet sich viel Harz, aber keine Säure.

Durch Behandeln von Campher mit Natrium und Kohlensäure erhielt B a ubig n y *) Borneol und Camphocarbonsäure. Wir haben das Absinthol genau nach Baubi g n y's Vorschrift behandelt, dabei aber weder eine Camphocarbonsäure, noch einen Körper von der Zusammensetzung des Borneols erhalten. Das Natrium wirkte zwar ein, beim Zersetzen des Productes mit Wasser ging aber die Hauptmenge bei $195^{\circ}$ über und zeigte die Zusammensetzung des angewandten Absinthols. Die niedriger und höher siedenden Antheile des Reactionsproductes waren ganz unbeträchtlich.

Besitzt dernnach das Absinthol die Zusammensetzung des Camphers, so bildet es dennoch kein Isomeres desselben im engeren Sinne : es gehört offenbar einer ganz anderen Gruppe von Verbindungen an.

Die höher siedenden Antheile des Wermuthöles sind stark gefärbt, namentlich besitzen die Dämpfe der zwischen $270^{\circ}$ und $300^{\circ}$ siedenden Portionen eine prächtige, tief indigblaue Farbe. Darüber hinaus werden die Dämpfe violett und zuletzt farblos, indem ein dunkler Theer in der Retorte zurückbleibt. Sämmtliche Antheile reagirten stark sauer und wurden daher wiederholt mit Aetzkali gewaschen. Ein fortgesetztes Fractioniren führte zu keinem constanten Siedepunkt. Eine Portion des blauen Oeles, das wegen beigemengten Wassers einige Zeit mit Chlorcalciumstücken an einen warmen Ort gestellt war, hatte seine blaue Farbe verloren und war oliven-

*) Jahresbericht für Chemie u. s. w. für $1868,497$. 
grün geworden. Die Analysen zeigen deutlich, dâfs das untersuchte Oel ein Gemenge war.

1. Siedep. $198-201^{\circ}$. Substanz 0,$3585 ; \mathrm{CO}_{2} 0,9634 ; \mathrm{H}_{2} \mathrm{O} 0,3608$.

2. $\gg 200-210^{\circ}$. $\quad 0,3892 ; n 1,1017 ; \quad 0,3965$.

3. $n \quad 210-255^{\circ}$. $n \quad 0,4179 ; n 1,2703 ; n 0,4253$.

4. $n \quad 255-270^{\circ}$. $n \quad 0,3854 ; \quad n 1,1862 ; n 0,3907$.

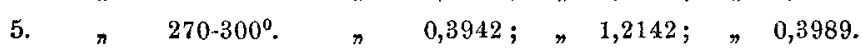

6. $\quad 300-320^{\circ}$. $\quad 0,4231 ; \quad 1,3095 ; \quad 0,4118$.

7. $\Rightarrow$ über $320^{\circ}$. $\gg 0,3556 ;, 1,1116 ; \Rightarrow 0,3518$.

\begin{tabular}{|c|c|c|c|c|c|c|c|c|}
\hline \multirow{2}{*}{\multicolumn{2}{|c|}{$\begin{array}{c}\text { Berechnet } \\
\mathrm{C}_{10} \mathrm{H}_{16} \mathrm{O}\end{array}$}} & \multicolumn{7}{|c|}{ Gefunden } \\
\hline & & $\begin{array}{c}1 . \\
198-201\end{array}$ & $\begin{array}{c}2 \\
1^{0} 200-210^{\circ}\end{array}$ & $\begin{array}{c}3 . \\
210-25\end{array}$ & $\begin{array}{c}4 . \\
255-270\end{array}$ & $\begin{array}{c}5 \\
270-30\end{array}$ & $\begin{array}{c}6 . \\
300-32\end{array}$ & $\begin{array}{c}7 . \\
\text { üb. } 320^{\circ}\end{array}$ \\
\hline C & 79,0 & 75,6 & 77,2 & 82,9 & 83,9 & 86,3 & 84,8 & 85,3 \\
\hline \multirow[t]{4}{*}{$\mathrm{H}$} & 10,5 & 11,2 & 11,3 & 11,3 & 11,3 & 11,2 & 10,8 & 11,0 \\
\hline & & & \multicolumn{2}{|c|}{$\begin{array}{c}\text { Berechnet } \\
\mathrm{C}_{10} \mathrm{H}_{16}\end{array}$} & \multicolumn{2}{|c|}{$\begin{array}{c}\text { Berechnet } \\
\mathrm{C}_{16} \mathrm{H}_{26} \mathrm{O} \\
(\text { Piesse) }\end{array}$} & & \\
\hline & & & \multicolumn{2}{|c|}{88,6} & \multicolumn{2}{|r|}{82,1} & & \\
\hline & & & \multicolumn{2}{|c|}{11,4} & & 11,1 . & & \\
\hline
\end{tabular}

Unsere Beobachtungen stimmen vollkommen mit denjenigen Kachler's ") über das blaue Kamillenöl überein, so dafs die Angabe Gladstone's über die Identität beider Oele keinem Zweifel unterliegt. Damit ist aber auch die Identität dieses Körpers mit dem blauen Destillationsproduct des Galbanumbarzes von Mo fs mer*\%) erwiesen. Unsere Analysen

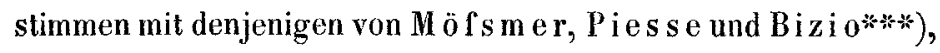
der ebenfalls das blaue Kamillenöl früher untersucht hatte, völlig überein. K a $\mathrm{chler}$, dem eine grofse Menge von völlig reinem Kamillenöl zur Verfügung stand, hat aber überzeugend nachgewiesen, dafs das blaue Oel ein Gemenge ist von einem hochsiedenden Terpen und einem Polymeren des Camphers $3\left(\mathrm{C}_{10} \mathrm{H}_{16} \mathrm{O}\right)$.

\footnotetext{
*) Berichte der deutschen chemischen Gesellschaft 4,36 .

**) Diese Annalen 119, 257.

***) Jahresbericht für Chemie u. s, w. für 1861, 681.

Annal. d. Ohem. u. Pharm. 170. Bd. 
Kachler fand, dafs das rectificirte Kamillenöl sauer reagirte und er isolirte aus dem zum Waschen benutzten Kali eine Säure von der Zusammensetzung der Caprinsäure. Durch das Behandeln der einzelnen Fractionen mit Kali konnten wir aus dem Wermuthöl eine sehr ansehnliche Menge Säure abscheiden. Wir säuerten die Kalilösungen mit Schwefelsäure an und schüttelten wiederholt mit Aether aus. Die rückständige Flüssigkeit anhaltend mit Wasser destillirt gab eine neue Menge Säure. Letztere erwies sich als nahezu reine Essigsäure. Dieselbe wurde nicht nur durch die gewöhnlichen Reactionen, sondern auch durch das charakteristische Barytsalz erkannt. Die concentrirte wässerige Lösung des Barytsalzes schied nämlich auf Zusatz von starkem Alkohol prächtige seideglänzende Nadeln aus.

0,7061 Grm. des bei $120^{\circ}$ getrockneten Salzes gaben 0,6435 $\mathrm{BaSO}_{4}$.

$\begin{array}{ccc} & \text { Berechnet } & \text { Gefunden } \\ \text { Essigsaurer Baryt Ba } & \mathbf{5 3 , 7} & 53,6 .\end{array}$

\section{Cymol aus Wermuthöl.}

Absinthol wurde mit dem halben Gewicht Schwefelphosphor zusammengebracht. Bei gelindem Erwärmen trat eine sehr heftige Reaction ein, nach deren Beendigung alles Flüchtige abdestillirt wurde. Das Destillat behandelten wir mit Natronlauge und dann wiederholt mit kleinen Mengen concentrirter Schwefelsäure. Um aber sicher alles Absinthol zu entfernen, haben wir das rohe Cymol noch ein zweites Mal mit einer kleineren Menge Schwefelphosphor behandelt und dann die Behandlung mit Natronlauge und Schwefelsäure wiederholt. Schliefslich wurde das Cymol drei Mal über Natrium rectificirt. Schon beim dritten Mal blieb das Natrium völlig blank und alles Cymol ging völlig constant bei $175^{\circ}$ über.

0,3421 Grm. gaben $0,3328 \mathrm{H}_{2} \mathrm{O}$ und $1,1133 \mathrm{CO}_{8}$. 
Beilstein u. Kupffer, über Wermuthöl.

$\begin{array}{lcc} & \text { Berechnet } & \text { Gefunden } \\ \mathrm{C}_{10} & 89,5 & 88,8 \\ \mathrm{H}_{14} & 10,5 & 10,8 .\end{array}$

Specifisches Gewicht des Cymols bei $0^{0}=\mathbf{0 , 8 7 0 7}$.

Cymolsulfosäure. Das Barytsalz, $\left(\mathrm{C}_{10} \mathrm{H}_{13} \mathrm{SO}_{3}\right)_{2} \mathrm{Ba}+3 \mathrm{H}_{2} \mathrm{O}$, bildete glänzende Schuppen, welche das Krystallwasser nicht über Schwefelsäure verloren.

0,390 Grm. gaben $0,0338 \mathrm{H}_{2} \mathrm{O}$ und $0,1465 \mathrm{BaSO}_{4}$.

15,8214 Grm. Lösung bei $15,8^{\circ}$ gaben $0,1831 \mathrm{BaSO}_{4}$.

$\begin{array}{ccc} & \text { Berechnet } & \text { Gefunden } \\ 3 \mathrm{H}_{\mathbf{2}} \mathrm{O} & 8,75 & 8,89 \\ \mathrm{Ba} & 24,3 & 24,2 .\end{array}$

100 Theile $\mathrm{H}_{2} \mathrm{O}$ lösen bei $15,8^{\circ} 2,877$ Th. wasserfreies Salz. Die Löslichkeit dieses Salzes ist etwas gröfser gefunden wie bei den Salzen aus Campher oder Kümmelöl (100:2,503 bei $16,2^{\circ}$ ), doch mufs bemerkt werden, dafs das Salz aus dem Absintholcymol etwas gelblich gefärbt war und daher die geringe Differenz durch eine Spur einer fremden Beimengung zu erklären ist.

Bleisalz. Im Aeufseren dem Salze aus anderen Cymolen völlig entsprechend.

0,9492 Grm. über Schwefelsäure getrocknet gaben $0,0736 \mathrm{H}_{2} \mathrm{O}$.

$$
\begin{array}{ccc} 
& \text { Berechnet } & \text { Gefunden } \\
3 \mathrm{H}_{\mathbf{2}} \mathrm{O} & 7,9 & 7,8 .
\end{array}
$$

Das Krystallwasser entweicht nicht im Exsiccator. Durch Kochen mit Wasser wird das Salz etwas zersetzt.

Kalksalz, $\left(\mathrm{C}_{10} \mathrm{H}_{13} \mathrm{SO}_{3}\right)_{2} \mathrm{Ca}+2 \mathrm{H}_{2} \mathrm{O}$. - Grofse glänzende Krystalle, in Wasser viel leichter löslich als das Barytsalz. Verliert über Schwefelsäure kein Wasser.

0,3395 Grm. gaben $0,0236 \mathrm{H}_{2} \mathrm{O}$.

$$
\begin{array}{ccc}
2 \mathrm{H}_{2} \mathrm{O} & \text { Berechnet } & \text { Gefunden } \\
7,2 & 7,0 .
\end{array}
$$

Dic Uebereinstimmung in dem Verhalten dieses Cymols mit demjenigen aus Campher und aus Kümmelöl ist demnach 
eine so vollkommene, dafs an der Identität derselben kein Zweifel bleiben kann. Eine weitere Bestätigung des Gesagten liefert die krystallographische Untersuchung des Kalksalzes von Herrn M. Jer ofejew.

Die Identität des Cymols von dreierlei verschiedenem Ursprung macht es wahrscheinlich, dafs auch das auf anderen Wegen erhaltene Cymol mit dem obigen gleich ist. Hierher gehört vor Allem das Cymol aus dem flüchtigen Oel des Wasserschierlings (Cicuta virosa), denn nach Trapp's Untersuchung *) ist dieses Oel vollkommen identisch mit dem Römisch-Kümmelöl. Dann das Cymol aus dem Oel von Ptychotis ajowan, das nach Hugo Müller**) mit dem Cymol aus Römisch-Kümmelöl völlig übereinstimmt. Endlich auch wohl das Cymol aus Terpentin- oder Citronenöl. Deville***) hat bereits durch Einwirkung feuchter Kohlensäure auf Terpentinöl bei Rothgluth Cymol erhalten. Einfacher gelangte C. G. Williams f) zu demselben Resultat, indem er Terpentinöl mit Brom behandelte und dann Natrium darauf einwirken liefs. Oppenheim t+), Barbier $+t+$ ) und $\mathrm{Ke}$ kulé*), welche sich in neuerer Zeit mit der Bildung von Cymol aus Terpentinöl beschäftigt haben, scheinen die älteren Versuche in gleicher Richtung übersehen zu haben. Oppenhei m beschreibt das Barytsalz seiner Sulfocymolsäure, übereinstimmend mit unseren Beobachtungen, als in Schuppen kry-

*) Diese Anualen 108, 386 .

**) Haines, Jahresber. für Chemie u. s. w. für 1856, 622; Stenhouse, diese Annalen 98, 307; H. Müller, Berichte der deutschen chemischen Gesellschaft $\mathbf{2}, 130$.

***) Gmelin's Handbuch 8, 176.

†) Jahresber, für Chemie u. s. w. für 1860, 495.

††) Berichte der deutschen chemischen Gesellschaft 5, 96, 628.

+†) Daselbst $5,215$.

*) Daselbst 6, 437 . 
stallisirend, mit 3 Mol. Krystallwasser. Auch gab sein Cymol mit Brom ein öliges Derivat.

Vor Kurzem hat auch Wrigh $t^{*}$ ) eine Untersuchung über Cymole veröffentlicht. Er untersuchte die Cymole aus Campher, Römisch-Kümmel-, Terpentin-, Muskatnufs- und Pomeranzenöl und hält dieselben sämmtlich für identisch. Leider lassen die bisher bekannt gewordenen kurzen Mittheilungen aus der Arbeit keinen Schlufs über die Ansicht Wright's zu. Wir glauben durch unsere Beobachiungen das Cymol in einer Weise charakterisirt zu haben, dafs es bei späteren Forschungen stets leicht möglich sein wird, sich über die Natur eines Cymols zu orientiren.

\section{Krystallographische Untersuchung des cymol- schwefelsauren Kalks;}

\section{von M. Jerofejew.}

(Hierzu Tafel III.)

Die Kalksalze, mit Material aus verschiedener Abstammung dargestellt, sind durchaus übereinstimmend.

$$
\begin{gathered}
\text { Krystallsystem : monoklinometrisch. } \\
\mathrm{a}: \mathrm{b}: \mathrm{c}=1,373958: 1: 1,124195 ; \\
\gamma=95^{\circ} 13^{\prime} 17^{\prime \prime} .
\end{gathered}
$$

Die Krystalle zeigen sämmtlich eine sehr vollkommene Spaltbarkeit nach dem Klino- und Orthopinakoïd $\mathrm{g}^{\prime}(010)$ und $h^{\prime}(100)$.

*) Berichte der deutschen chemischen Gesellschaft $\mathbf{6}, 455$. 